\title{
Effect of Iron and Zinc Enriched FYM on Growth, Yield and Quality of Wheat (Triticum aestivum L) in Salt Affected Soils of Gujarat
}

\author{
J.K. Malav ${ }^{*}$, N.N. Salvi ${ }^{1}$, J.K. Patel ${ }^{2}$, J.R. Jat ${ }^{3}$, S. Kumar ${ }^{3}$, \\ R.P. Pavaya ${ }^{1}$, B.T. Patel ${ }^{1}$ and V.R. Patel ${ }^{1}$ \\ ${ }^{1}$ Department of Agricultural Chemistry and Soil Science, C.P. College of Agriculture, \\ S.D. Agricultural University, Sardarkrushinagar-385 506 (Gujarat), India \\ ${ }^{2}$ Agricultural Research Station, S.D. Agricultural University, Adiya, Gujarat, India \\ ${ }^{3}$ Agroforestry Research Centre, S.D. Agricultural University, Sardarkrushinagar, \\ Gujarat, India
}

*Corresponding author

\begin{tabular}{|l|}
\hline Ke y w o r d s \\
Wheat, FYM, Zinc, \\
Iron, Enriched \\
\hline Article Info \\
\hline $\begin{array}{l}\text { Accepted: } \\
\text { 24 May } 2019 \\
\text { Available Online: } \\
\text { 10 June } 2019\end{array}$ \\
\hline
\end{tabular}

\section{Keywords}

Wheat, FYM, Zinc,

\section{A B S T R A C T}

A field experiment entitled "Effect of iron and zinc enriched FYM on growth, yield and quality of wheat in salt affected soils of Gujarat" was conducted during 2014-15 to 201516, 2016-17 and 2017-18 at Agricultural Research Station, Sardarkrushinagar Dantiwada Agricultural University, Adiya, Gujarat. The experiment encompassed eight treatments combinations viz., $\mathrm{T}_{1}$ : RDF (Based on STV), $\mathrm{T}_{2}: \mathrm{T}_{1}+1.0 \mathrm{t} \mathrm{FYM} \mathrm{ha}^{-1} ; \mathrm{T}_{3}: \mathrm{T}_{1}+1.5 \mathrm{~kg} \mathrm{Zn}$ $\mathrm{ha}^{-1} ; \mathrm{T}_{4}: \mathrm{T}_{1}+3.0 \mathrm{~kg} \mathrm{Fe} \mathrm{ha}^{-1} ; \mathrm{T}_{5}: \mathrm{T}_{1}+1.5 \mathrm{~kg} \mathrm{Zn} \mathrm{ha}^{-1}+3.0 \mathrm{~kg} \mathrm{Fe} \mathrm{ha}^{-1} ; \mathrm{T}_{6}: \mathrm{T}_{1}+0.5 \mathrm{t} \mathrm{FYM} \mathrm{ha}^{-1}$ enriched with $0.75 \mathrm{~kg} \mathrm{Zn} ; \mathrm{T}_{7}: \mathrm{T}_{1}+0.5 \mathrm{t} F \mathrm{FM} \mathrm{ha}^{-1}$ enriched with $1.5 \mathrm{~kg} \mathrm{Fe} ; \mathrm{T}_{8}: \mathrm{T}_{1}+0.5 \mathrm{t}$ FYM ha ${ }^{-1}$ enriched with $0.75 \mathrm{~kg} \mathrm{Zn}$ and $1.5 \mathrm{~kg}$ Fe. The experiment was laid out in randomized block design with four replications. The results reveal that application of recommended dose of fertilizer (120-60-00 NPK kg ha ${ }^{-1}$ ) on the basis of STV + 0.5 t FYM $\mathrm{ha}^{-1}$ enriched with $0.75 \mathrm{~kg} \mathrm{Zn}$ and $1.5 \mathrm{~kg} \mathrm{Fe}\left(\mathrm{T}_{8}\right)$ recorded significantly higher effective tillers per plant, length of spike, no. of spikes per plant, no. of seeds per spike, plant height, grain and straw yields of wheat over $T_{1}$ (Recommended dose of fertilizer on the basis of STV only) during all the individual years as well as on pooled basis. The farmers of North Gujarat Agroclimatic zone having salt affected soil and interested to grow wheat are advised to apply recommended dose of fertilizer on the basis of STV + 0.5 t FYM ha ${ }^{-1}$ enriched with $0.75 \mathrm{~kg} \mathrm{Zn}$ and $1.5 \mathrm{~kg}$ Fe for getting higher yield and net returns.

\section{Introduction}

Wheat is one of the first cereals known to be grown and its ability to self pollinate greatly facilitated the selection of many distinct domesticated varieties. Wheat is the most important grain of trade for human consumption. It is produced in a vast range of environments from central Russia to the great India and Chinese river valleys and across the Great Plains and pampas of Americas. In India wheat is the second most important food crop after rice, both in terms of area and production. Wheat production of India is 
98.38 million tonne from 30.59 million hectares with productivity of 3.22 tonne per hectare during 2016-17. It accounts for about 36 percent of country's total food grain production as per the fourth advance estimate (Anonymous, 2017).

Zinc and iron deficiencies are welldocumented public health issue affecting nearly half of the world population especially in developing countries like India. Zinc and iron deficiencies are the common micronutrient deficiencies in light textured soils of North Gujarat limiting both crop production and nutritional quality. Further, very low concentrations and poor bioavailability of $\mathrm{Zn}$ and $\mathrm{Fe}$ in the commonly used cereals aggravated the micronutrient deficiencies. Breeding new cereal genotypes with high genetic capacity for grain accumulation of micronutrients is widely accepted and most sustainable solution to the problem. However, the breeding approach is a long-term process and may be affected from very low chemical solubility of $\mathrm{Zn}$ and $\mathrm{Fe}$ in soils due to high $\mathrm{pH}$ and low organic matter (Cakmak, 2008). Therefore, agronomy-related approaches offer short-term and complementary solutions to the $\mathrm{Zn}$ and $\mathrm{Fe}$ deficiency in crop production and human health. Soil amendments contributing to solubility of $\mathrm{Zn}$ and Fe in soil solution, cereallegume intercropping systems, and soil and foliar application of micronutrient-containing fertilizers are well-documented agronomic tools which contribute to root uptake, shoot and grain accumulation of $\mathrm{Fe}$ and $\mathrm{Zn}$. Addition of organic material had beneficial effect on crop growth, productivity by sustaining soil health. Mixing inorganic salts of micronutrients with different organic materials can enhance the efficacy of micronutrients. On decomposition of organic manures numerous compounds like humic acid and fulvic acid and biological substances like organic acids, amino acids and polyphenols are produced which act as chelating agents that form stable complexes with native micronutrients and also prevent added inorganic micronutrients from precipitation, fixation, oxidation and leaching resulted in improvement in efficiency of applied micronutrients. The enrichment of organics with micronutrients not only improve the quality of organics but also reduced the quantity of both inorganic chemicals and as well as quantity of organics. It is reported that addition of enriched organics in lower quantities had similar effects on soil properties to that of high quantity (without enrichment). The enriched organics are expected to provide beneficial effect on plant growth for longer time. The information on $\mathrm{Fe}$ and $\mathrm{Zn}$ enriched organics are lacking on $\mathrm{Fe}$ and $\mathrm{Zn}$ deficient soils of North Gujarat where wheat crop is mainly grown as cereal crop.

The implementation of agronomic biofortification of cereal crops with iron and zinc appear to be a rapid and simple solution to the deficiency of these elements in the soils and plants. Zinc plays an important role in carbohydrate metabolism, detoxification of super oxide radical and imparts resistance to disease in plants. Since $\mathrm{Zn}$ is associated with enzymes, its deficiency leads to several disorders in plants. $\mathrm{Zn}$ deficiency has received great attention in India, because nearly half of the Indian soils are poor in available $\mathrm{Zn}$ content. (Shivay et al., 2014). Zinc is mainly localized and concentrated in the aleurone and embryo parts of wheat grain. Zinc concentration of the endosperm (white flour) is very small. Wheat grain is consumed after milling, which removes the Zn-rich parts and leaves just the $\mathrm{Zn}$-poor endosperm behind (Cakmak, 2017).

Iron plays a key role in the synthesis of chlorophyll, carbohydrate production, cell respiration, chemical reduction of nitrate and 
sulphate, and in $\mathrm{N}$ assimilation. The $\mathrm{Fe}$ is mainly involved in biochemical processes which are mostly enzymatic oxidationreduction reactions in plants. With the progress of time and advancement in agricultural technology, there is a need to maximize awareness regarding healthy nutrition at both national and international level. There is a paucity of research data on agronomic biofortification of new wheat varieties using fertilizer strategies for improvement in yield and grain quality in Gujarat (India). Keeping in view the above facts, this study was initiated to assess the effect of iron and zinc enriched FYM on growth, yield and quality of wheat in salt affected soils of Gujarat.

\section{Materials and Methods}

A field experiment was conducted during 2014-15 to 2015-16, 2016-17 and 2017-18 at Agricultural Research Station, Sardarkrushinagar Dantiwada Agricultural University, Adiya, Gujarat. This experiment take in eight treatment combinations viz., $\mathrm{T}_{1}$ : RDF (Based on STV), T ${ }_{2} \mathrm{~T}_{1}+1.0 \mathrm{t} \mathrm{FYM} \mathrm{ha}^{-1}$; $\mathrm{T}_{3}: \mathrm{T}_{1}+1.5 \mathrm{~kg} \mathrm{Zn} \mathrm{ha}^{-1} ; \mathrm{T}_{4}: \mathrm{T}_{1}+3.0 \mathrm{~kg} \mathrm{Fe} \mathrm{ha}^{-1}$; $\mathrm{T}_{5}: \mathrm{T}_{1}+1.5 \mathrm{~kg} \mathrm{Zn} \mathrm{ha}{ }^{-1}+3.0 \mathrm{~kg} \mathrm{Fe} \mathrm{ha}{ }^{-1} ; \mathrm{T}_{6}: \mathrm{T}_{1}$ +0.5 t FYM ha $^{-1}$ enriched with $0.75 \mathrm{~kg} \mathrm{Zn}$; $\mathrm{T}_{7}: \mathrm{T}_{1}+0.5$ t FYM ha ${ }^{-1}$ enriched with $1.5 \mathrm{~kg}$ Fe; $\mathrm{T}_{8}: \mathrm{T}_{1}+0.5$ t FYM ha ${ }^{-1}$ enriched with 0.75 $\mathrm{kg} \mathrm{Zn}$ and $1.5 \mathrm{~kg} \mathrm{Fe}$ in randomized block design, which was replicated four times and wheat variety Raj-3077 was sown.

The soil of the experimental field was loamy sand in texture, alkaline in reaction and soluble salt content under unsafe limit. It was low in organic carbon, medium in available $\mathrm{P}_{2} \mathrm{O}_{5}$ and medium to high in available $\mathrm{K}_{2} \mathrm{O}$ and DTPA extractable $\mathrm{Zn}$ as well as in DTPA extractable Fe (Table 1). The data of seed yield and straw yield recorded from net plot and converted on hectare basis. The collected data for various parameters were statistically analyzed using Fishers' analysis of variance (ANOVA) technique and the treatments were compared at $5 \%$ level of significance.

\section{Methodology for the enrichment of FYM with $\mathrm{Fe}$ and $\mathrm{Zn}$}

The enrichment process was started 45 days before their use.

Required quantity of $\mathrm{ZnSO}_{4} \cdot 7 \mathrm{H}_{2} \mathrm{O}$ or $\mathrm{FeSO}_{4} .7 \mathrm{H}_{2} \mathrm{O}$ (as per treatment i.e. 0.75 \& $1.5 \mathrm{~kg} \mathrm{Zn} / \mathrm{ha}$ and $1.5 \& 3.0 \mathrm{~kg} \mathrm{Fe} / \mathrm{ha}$ ) was thoroughly mixed with $500 \mathrm{~kg}$ FYM/ha.

Cow dung slurry @ 1\% was added to boost up the microbial activities for enhancement of natural process of composting to fix the externally added inorganic $\mathrm{Fe}$ and $\mathrm{Zn}$ in to organically bound and naturally chelated form of Fe and $\mathrm{Zn}$.

It was filled in the pre-dug polythene lined pits of $1.5 \times 1.5 \times 1.5 \mathrm{~m}^{3}$ size.

About 75 percent moisture of this mixture was maintained after mixing of $\mathrm{ZnSO}_{4} .7 \mathrm{H}_{2} \mathrm{O}$ or $\mathrm{FeSO}_{4} .7 \mathrm{H}_{2} \mathrm{O}$ with FYM and addition of cow dung slurry.

The pit was covered with polythene sheet and allowed for decomposition. The mixture was turned over periodically (Weekly) and moisture loss was maintained. The enrichment process was considered as completed after 5 to 6 weeks.

\section{Results and Discussion}

\section{Growth and quality characters}

The results presented in Table 2 to 7 reveal that application of recommended dose of fertilizer (120-60-00 NPK $\mathrm{kg} \mathrm{ha}^{-1}$ ) on the basis of STV + $0.5 \mathrm{t} \mathrm{FYM} \mathrm{ha}^{-1}$ enriched with $0.75 \mathrm{~kg} \mathrm{Zn}$ and $1.5 \mathrm{~kg} \mathrm{Fe}\left(\mathrm{T}_{8}\right)$ recorded significantly higher effective tillers per plant (6.50, 6.50, 6.55, 6.86 and 6.60), length of 
spike $(9.23,8.74,9.14,9.78$ and $9.22 \mathrm{~cm})$, no. of spike per plant $(6.35,5.97,6.17,5.92$ and $6.10)$, no. of seeds per spike $(34.14,35.96$, $35.25,36.02$ and 35.34), plant height and $(75.78,74.85,74.85,79.74$ and $75.56 \mathrm{~cm})$ of wheat over $\mathrm{T}_{1}$ (Recommended dose of fertilizer on the basis of STV only) during all the individual years as well as on pooled basis. But, it was at par with $\mathrm{T}_{5}$ on pooled basis in case of effective tillers per plant (6.46), no. of spikes per plant (5.94), no. of seeds per spike (34.99) and plant height $(74.18 \mathrm{~cm})$. The better efficiency of organic matter might be due to the fact that the organic manure especially FYM would have provided micronutrient at optimum level which play important role in chlorophyll formation which increase rate of photosynthesis and ultimately growth of the plant. These results are in accordance with the findings of Navrang and Tomar (2016). Beneficial effect of $\mathrm{Zn}$ through soil incorporation and foliar application of $\mathrm{FeSO}_{4}$ at tillering stage to affect an increase in growth characteristics in this study may probably be assigned to harmonious plant physiology as stated by Gul et al., (2011).

Table.1 Physicochemical properties of the experimental soil

\begin{tabular}{|c|c|c|c|c|c|}
\hline \multirow[t]{2}{*}{ Sr. No. } & \multirow[t]{2}{*}{ Parameters } & \multicolumn{4}{|c|}{ Years } \\
\hline & & 2014-15 & 2015-16 & 2016-17 & 2017-18 \\
\hline 1. & $\mathrm{pH}$ & 7.70 & 8.12 & 7.64 & 6.98 \\
\hline 2. & $\mathrm{EC}\left(\mathrm{dSm}^{-1}\right)$ & 1.10 & 1.18 & 1.35 & 1.45 \\
\hline 3. & $\mathrm{OC} \%$ & 0.31 & 0.25 & 0.38 & 0.26 \\
\hline 4. & Available $\mathrm{P}_{2} \mathrm{O}_{5}(\mathrm{~kg} / \mathrm{ha})$ & 45.87 & 41.25 & 43.25 & 46.58 \\
\hline 5. & Available $\mathrm{K}_{2} \mathrm{O}(\mathrm{kg} / \mathrm{ha})$ & 241.25 & 285.54 & 356.14 & 266.14 \\
\hline 6. & DTPA extractable Fe $\left(\mathrm{mg} \mathrm{kg}^{-1}\right)$ & 3.78 & 3.95 & 3.51 & 2.25 \\
\hline 7. & DTPA extractable $\mathrm{Zn}\left(\mathrm{mg} \mathrm{kg}^{-1}\right)$ & 0.89 & 1.08 & 0.89 & 0.76 \\
\hline & RDF Based on STV & 140:60:0 & 150:60:0 & 140:60:0 & 140:60:0 \\
\hline
\end{tabular}

Table.2 Effect of iron and zinc enriched with FYM and without enriched FYM on no. of effective tillers per plant of wheat

\begin{tabular}{|c|c|c|c|c|c|}
\hline \multirow[t]{2}{*}{ Treatments } & \multicolumn{5}{|c|}{ No. of effective tillers per plant } \\
\hline & 2014-15 & 2015-16 & 2016-17 & 2017-18 & Pooled \\
\hline$T_{1}$ RDF (Based on STV) & 4.75 & 5.00 & 4.61 & 4.88 & 4.81 \\
\hline $\mathrm{T}_{2} \mathrm{~T}_{1}+1.0 \mathrm{t}$ FYM ha $\mathrm{h}^{-1}$ & 5.01 & 5.00 & 4.96 & 5.46 & 5.11 \\
\hline $\mathrm{T}_{3} \mathrm{~T}_{1}+1.5 \mathrm{~kg} \mathrm{Zn} \mathrm{ha}^{-1}$ & 5.01 & 5.00 & 5.17 & 5.43 & 5.15 \\
\hline $\mathrm{T}_{4} \mathrm{~T}_{1}+3.0 \mathrm{~kg} \mathrm{Fe} \mathrm{ha}{ }^{-1}$ & 5.51 & 5.50 & 5.01 & 5.57 & 5.40 \\
\hline $\mathrm{T}_{5} \mathrm{~T}_{1}+1.5 \mathrm{~kg} \mathrm{Zn} \mathrm{ha}^{-1}+3.0 \mathrm{~kg} \mathrm{Fe} \mathrm{ha}^{-1}$ & 6.25 & 6.50 & 6.42 & 6.66 & 6.46 \\
\hline $\mathrm{T}_{6} \mathrm{~T}_{1+} 0.5 \mathrm{t}$ FYM ha ${ }^{-1}$ En. with $0.75 \mathrm{~kg} \mathrm{Zn}$ & 5.25 & 5.50 & 5.34 & 5.48 & 5.39 \\
\hline $\mathrm{T}_{7} \mathrm{~T}_{1+} 0.5 \mathrm{t}$ FYM ha ${ }^{-1} \mathrm{En}$. with $1.5 \mathrm{~kg} \mathrm{Fe}$ & 5.25 & 5.50 & 5.61 & 5.73 & 5.52 \\
\hline $\mathrm{T}_{8} \mathrm{~T}_{1+} 0.5 \mathrm{t} \mathrm{FYM} \mathrm{ha}{ }^{-1}$ En. with $0.75 \mathrm{~kg} \mathrm{Zn}$ and $1.5 \mathrm{~kg} \mathrm{Fe}$ & 6.50 & 6.50 & 6.55 & 6.86 & 6.60 \\
\hline S.Em. \pm & 0.30 & 0.40 & 0.14 & 0.19 & 0.14 \\
\hline C.D. at $5 \%$ & 0.89 & NS & 0.42 & 0.55 & 0.39 \\
\hline C.V. \% & 11.06 & 14.48 & 5.22 & 6.52 & 10.00 \\
\hline \multicolumn{6}{|l|}{ Interactions } \\
\hline $\mathbf{Y} \times \mathbf{T}$ & \multicolumn{5}{|c|}{ NS } \\
\hline
\end{tabular}


Table.3 Effect of iron and zinc enriched with FYM and without enriched FYM on length of spike $(\mathrm{cm})$ of wheat

\begin{tabular}{|c|c|c|c|c|c|}
\hline \multirow[t]{2}{*}{ Treatments } & \multicolumn{5}{|c|}{ Length of spike (cm) } \\
\hline & 2014-15 & 2015-16 & 2016-17 & 2017-18 & Pooled \\
\hline$T_{1}$ RDF (Based on STV) & 6.53 & 7.01 & 6.86 & 7.19 & 6.90 \\
\hline $\mathrm{T}_{2} \mathrm{~T}_{1}+1.0 \mathrm{t}$ FYM ha ${ }^{-1}$ & 7.38 & 7.55 & 7.26 & 7.54 & 7.43 \\
\hline $\mathrm{T}_{3} \mathrm{~T}_{1}+1.5 \mathrm{~kg} \mathrm{Zn} \mathrm{ha}^{-1}$ & 7.45 & 7.60 & 7.88 & 7.82 & 7.69 \\
\hline $\mathrm{T}_{4} \mathrm{~T}_{1}+3.0 \mathrm{~kg} \mathrm{Fe} \mathrm{ha}^{-1}$ & 7.71 & 7.76 & 7.88 & 7.84 & 7.80 \\
\hline$T_{5} T_{1}+1.5 \mathrm{~kg} \mathrm{Zn} \mathrm{ha}^{-1}+3.0 \mathrm{~kg} \mathrm{Fe} \mathrm{ha}^{-1}$ & 8.45 & 8.24 & 8.48 & 9.49 & 8.66 \\
\hline $\mathrm{T}_{6} \mathrm{~T}_{1}+0.5 \mathrm{t} \mathrm{FYM} \mathrm{ha}^{-1}$ En. with $0.75 \mathrm{~kg} \mathrm{Zn}$ & 7.80 & 7.82 & 7.53 & 8.82 & 7.99 \\
\hline $\mathrm{T}_{7} \mathrm{~T}_{1}+0.5 \mathrm{t} \mathrm{FYM} \mathrm{ha}{ }^{-1} \mathrm{En}$. with $1.5 \mathrm{~kg} \mathrm{Fe}$ & 7.98 & 7.94 & 8.20 & 8.77 & 8.22 \\
\hline $\mathrm{T}_{8} \mathrm{~T}_{1}+0.5 \mathrm{t} \mathrm{FYM} \mathrm{ha}^{-1} \mathrm{En}$. with $0.75 \mathrm{~kg} \mathrm{Zn}$ and $1.5 \mathrm{~kg} \mathrm{Fe}$ & 9.23 & 8.74 & 9.14 & 9.78 & 9.22 \\
\hline S.Em. \pm & 0.25 & 0.20 & 0.35 & 0.16 & 0.13 \\
\hline C.D. at $5 \%$ & 0.73 & 0.60 & 1.04 & 0.47 & 0.35 \\
\hline C.V. \% & 6.35 & 5.20 & 8.91 & 3.83 & 6.29 \\
\hline \multicolumn{6}{|l|}{ Interactions } \\
\hline $\mathbf{Y} \times \mathbf{T}$ & \multicolumn{5}{|c|}{ NS } \\
\hline
\end{tabular}

Table.4 Effect of iron and zinc enriched with FYM and without enriched FYM on No. of spikes per plant of wheat

\begin{tabular}{|c|c|c|c|c|c|}
\hline \multirow[t]{2}{*}{ Treatments } & \multicolumn{5}{|c|}{ No. of spikes per plant } \\
\hline & 2014-15 & 2015-16 & 2016-17 & 2017-18 & Pooled \\
\hline$T_{1}$ RDF (Based on STV) & 4.94 & 4.56 & 4.69 & 4.69 & 4.72 \\
\hline $\mathrm{T}_{2} \mathrm{~T}_{1}+1.0 \mathrm{t}$ FYM ha ${ }^{-1}$ & 5.24 & 4.86 & 5.16 & 5.16 & 5.10 \\
\hline $\mathrm{T}_{3} \mathrm{~T}_{1}+1.5 \mathrm{~kg} \mathrm{Zn} \mathrm{ha}^{-1}$ & 5.35 & 4.97 & 4.45 & 4.45 & 4.80 \\
\hline $\mathrm{T}_{4} \mathrm{~T}_{1}+3.0 \mathrm{~kg} \mathrm{Fe} \mathrm{ha}^{-1}$ & 5.22 & 4.84 & 5.06 & 5.06 & 5.04 \\
\hline $\mathrm{T}_{5} \mathrm{~T}_{1}+1.5 \mathrm{~kg} \mathrm{Zn} \mathrm{ha}^{-1}+3.0 \mathrm{~kg} \mathrm{Fe} \mathrm{ha}^{-1}$ & 6.27 & 5.89 & 5.80 & 5.80 & 5.94 \\
\hline $\mathrm{T}_{6} \mathrm{~T}_{1+} 0.5$ t FYM ha ${ }^{-1}$ En. with $0.75 \mathrm{~kg} \mathrm{Zn}$ & 5.70 & 5.31 & 4.73 & 4.73 & 5.11 \\
\hline $\mathrm{T}_{7} \mathrm{~T}_{1+} 0.5 \mathrm{t}$ FYM ha ${ }^{-1}$ En. with $1.5 \mathrm{~kg} \mathrm{Fe}$ & 5.62 & 5.23 & 5.50 & 5.50 & 5.46 \\
\hline $\mathrm{T}_{8} \mathrm{~T}_{1+} 0.5 \mathrm{t}$ FYM ha ${ }^{-1} \mathrm{En}$. with $0.75 \mathrm{~kg} \mathrm{Zn}$ and $1.5 \mathrm{~kg} \mathrm{Fe}$ & 6.35 & 5.97 & 6.17 & 5.92 & 6.10 \\
\hline S.Em. \pm & 0.26 & 0.18 & 0.29 & 0.27 & 0.13 \\
\hline C.D. at $5 \%$ & 0.75 & 0.53 & 0.86 & 0.81 & 0.36 \\
\hline C.V. \% & 9.19 & 6.91 & 11.20 & 10.61 & 9.61 \\
\hline \multicolumn{6}{|l|}{ Interactions } \\
\hline $\mathbf{Y} \times \mathbf{T}$ & \multicolumn{5}{|c|}{ NS } \\
\hline
\end{tabular}


Table.5 Effect of iron and zinc enriched with FYM and without enriched FYM on No. of seeds per spike of wheat

\begin{tabular}{|c|c|c|c|c|c|}
\hline \multirow[t]{2}{*}{ Treatments } & \multicolumn{5}{|c|}{ No. of seeds per spike } \\
\hline & 2014-15 & 2015-16 & 2016-17 & 2017-18 & Pooled \\
\hline$T_{1}$ RDF (Based on STV) & 25.75 & 27.57 & 28.58 & 30.70 & 28.15 \\
\hline $\mathrm{T}_{2} \mathrm{~T}_{1}+1.0 \mathrm{t} \mathrm{FYM} \mathrm{ha}^{-1}$ & 27.68 & 29.50 & 28.94 & 31.11 & 29.30 \\
\hline$T_{3} T_{1}+1.5 \mathrm{~kg} \mathrm{Zn} \mathrm{ha}^{-1}$ & 29.69 & 31.51 & 30.90 & 33.05 & 31.29 \\
\hline $\mathrm{T}_{4} \mathrm{~T}_{1}+3.0 \mathrm{~kg} \mathrm{Fe} \mathrm{ha}^{-1}$ & 30.80 & 32.62 & 29.06 & 33.28 & 31.44 \\
\hline$T_{5} T_{1}+1.5 \mathrm{~kg} \mathrm{Zn} \mathrm{ha}^{-1}+3.0 \mathrm{~kg} \mathrm{Fe} \mathrm{ha}^{-1}$ & 33.78 & 35.60 & 34.75 & 35.85 & 34.99 \\
\hline $\mathrm{T}_{6} \mathrm{~T}_{1+} 0.5 \mathrm{t}$ FYM ha ${ }^{-1}$ En. with $0.75 \mathrm{~kg} \mathrm{Zn}$ & 32.05 & 33.87 & 32.15 & 34.02 & 33.02 \\
\hline $\mathrm{T}_{7} \mathrm{~T}_{1+} 0.5 \mathrm{t}$ FYM ha ${ }^{-1}$ En. with $1.5 \mathrm{~kg} \mathrm{Fe}$ & 32.31 & 34.13 & 31.40 & 35.11 & 33.24 \\
\hline $\mathrm{T}_{8} \mathrm{~T}_{1+} 0.5 \mathrm{t} \mathrm{FYM} \mathrm{ha}{ }^{-1} \mathrm{En}$. with $0.75 \mathrm{~kg} \mathrm{Zn}$ and $1.5 \mathrm{~kg} \mathrm{Fe}$ & 34.14 & 35.96 & 35.25 & 36.02 & 35.34 \\
\hline S.Em. \pm & 1.85 & 0.89 & 1.36 & 0.91 & 0.66 \\
\hline C.D. at $5 \%$ & 5.43 & 2.61 & 4.00 & 2.68 & 1.85 \\
\hline C.V. \% & 12.00 & 5.45 & 8.67 & 5.42 & 8.17 \\
\hline \multicolumn{6}{|l|}{ Interactions } \\
\hline $\mathbf{Y} \times \mathbf{T}$ & \multicolumn{5}{|c|}{ NS } \\
\hline
\end{tabular}

Table.6 Effect of iron and zinc enriched with FYM and without enriched FYM on plant height (cm) at harvest of wheat

\begin{tabular}{|c|c|c|c|c|c|}
\hline \multirow[t]{2}{*}{ Treatments } & \multicolumn{5}{|c|}{ Plant height (cm) } \\
\hline & 2014-15 & 2015-16 & 2016-17 & 2017-18 & Pooled \\
\hline $\mathrm{T}_{1}$ RDF (Based on STV) & 70.78 & 64.50 & 64.50 & 65.61 & 66.35 \\
\hline $\mathrm{T}_{2} \mathrm{~T}_{1}+1.0 \mathrm{t}$ FYM ha ${ }^{-1}$ & 72.02 & 69.25 & 69.25 & 67.24 & 69.44 \\
\hline $\mathrm{T}_{3} \mathrm{~T}_{1}+1.5 \mathrm{~kg} \mathrm{Zn} \mathrm{ha}^{-1}$ & 72.28 & 71.85 & 71.85 & 71.95 & 71.98 \\
\hline $\mathrm{T}_{4} \mathrm{~T}_{1}+3.0 \mathrm{~kg} \mathrm{Fe} \mathrm{ha}^{-1}$ & 69.77 & 69.88 & 69.88 & 70.84 & 70.09 \\
\hline $\mathrm{T}_{5} \mathrm{~T}_{1}+1.5 \mathrm{~kg} \mathrm{Zn} \mathrm{ha}^{-1}+3.0 \mathrm{~kg} \mathrm{Fe} \mathrm{ha}^{-1}$ & 75.27 & 73.23 & 73.23 & 75.02 & 74.18 \\
\hline $\mathrm{T}_{6} \mathrm{~T}_{1+} 0.5 \mathrm{t} \mathrm{FYM} \mathrm{ha}^{-1}$ En. with $0.75 \mathrm{~kg} \mathrm{Zn}$ & 74.03 & 70.50 & 70.50 & 70.91 & 71.48 \\
\hline $\mathrm{T}_{7} \mathrm{~T}_{1+} 0.5 \mathrm{t}$ FYM ha ${ }^{-1}$ En. with $1.5 \mathrm{~kg} \mathrm{Fe}$ & 72.27 & 70.63 & 70.63 & 71.30 & 71.20 \\
\hline $\mathrm{T}_{8} \mathrm{~T}_{1+} 0.5 \mathrm{t}$ FYM ha ${ }^{-1}$ En. with $0.75 \mathrm{~kg} \mathrm{Zn}$ and $1.5 \mathrm{~kg} \mathrm{Fe}$ & 75.78 & 74.85 & 74.85 & 76.74 & 75.56 \\
\hline S.Em. \pm & 1.32 & 1.46 & 1.46 & 1.66 & 0.74 \\
\hline C.D. at $5 \%$ & 3.90 & 4.30 & 4.30 & 4.89 & 2.09 \\
\hline C.V. \% & 3.64 & 4.15 & 4.15 & 4.67 & 4.16 \\
\hline \multicolumn{6}{|l|}{ Interactions } \\
\hline $\mathbf{Y} \mathbf{x} \mathbf{T}$ & \multicolumn{5}{|c|}{ NS } \\
\hline
\end{tabular}


Table.7 Effect of iron and zinc enriched with FYM and without enriched FYM on protein content $(\%)$ of wheat

\begin{tabular}{|c|c|c|c|c|c|}
\hline \multirow[t]{2}{*}{ Treatments } & \multicolumn{5}{|c|}{ Protein content $(\%)$} \\
\hline & 2014-15 & 2015-16 & 2016-17 & 2017-18 & Pooled \\
\hline$T_{1}$ RDF (Based on STV) & 12.41 & 12.08 & 11.92 & 12.90 & 12.33 \\
\hline $\mathrm{T}_{2} \mathrm{~T}_{1}+1.0 \mathrm{t} \mathrm{FYM} \mathrm{ha}^{-1}$ & 12.65 & 12.22 & 12.21 & 13.52 & 12.65 \\
\hline $\mathrm{T}_{3} \mathrm{~T}_{1}+1.5 \mathrm{~kg} \mathrm{Zn} \mathrm{ha}^{-1}$ & 12.87 & 12.42 & 12.44 & 13.53 & 12.81 \\
\hline $\mathrm{T}_{4} \mathrm{~T}_{1}+3.0 \mathrm{~kg} \mathrm{Fe} \mathrm{ha}^{-1}$ & 12.87 & 13.16 & 13.82 & 13.50 & 13.34 \\
\hline $\mathrm{T}_{5} \mathrm{~T}_{1}+1.5 \mathrm{~kg} \mathrm{Zn} \mathrm{ha}^{-1}+3.0 \mathrm{~kg} \mathrm{Fe} \mathrm{ha}^{-1}$ & 13.34 & 12.99 & 13.27 & 14.87 & 13.62 \\
\hline $\mathrm{T}_{6} \mathrm{~T}_{1+} 0.5$ t FYM ha ${ }^{-1}$ En. with $0.75 \mathrm{~kg} \mathrm{Zn}$ & 13.01 & 12.60 & 13.60 & 14.17 & 13.34 \\
\hline $\mathrm{T}_{7} \mathrm{~T}_{1+} 0.5 \mathrm{t}$ FYM ha ${ }^{-1}$ En. with $1.5 \mathrm{~kg} \mathrm{Fe}$ & 13.02 & 13.67 & 14.03 & 14.11 & 13.71 \\
\hline $\mathrm{T}_{8} \mathrm{~T}_{1}+0.5 \mathrm{t} \mathrm{FYM} \mathrm{ha}^{-1} \mathrm{En}$. with $0.75 \mathrm{~kg} \mathrm{Zn}$ and $1.5 \mathrm{~kg} \mathrm{Fe}$ & 13.17 & 13.32 & 14.27 & 14.64 & 13.85 \\
\hline S.Em. \pm & 0.43 & 0.56 & 0.77 & 0.70 & 0.31 \\
\hline C.D. at $5 \%$ & NS & NS & NS & NS & 0.88 \\
\hline C.V. \% & 6.63 & 8.78 & 11.60 & 10.06 & 9.50 \\
\hline \multicolumn{6}{|l|}{ Interactions } \\
\hline $\mathbf{Y} \times \mathbf{T}$ & \multicolumn{5}{|c|}{ NS } \\
\hline
\end{tabular}

Table.8 Effect of iron and zinc enriched with FYM and without enriched FYM on grain yield of wheat

\begin{tabular}{|c|c|c|c|c|c|}
\hline \multirow[t]{2}{*}{ Treatments } & \multicolumn{5}{|c|}{ Grain yield $\left(\mathrm{kg} \mathrm{ha}^{-1}\right)$} \\
\hline & 2014-15 & 2015-16 & 2016-17 & 2017-18 & Pooled \\
\hline$T_{1}$ RDF (Based on STV) & 3413 & 3563 & 4044 & 4104 & 3781 \\
\hline $\mathrm{T}_{2} \mathrm{~T}_{1}+1.0 \mathrm{t}$ FYM ha ${ }^{-1}$ & 3765 & 3881 & 4580 & 4712 & 4234 \\
\hline $\mathrm{T}_{3} \mathrm{~T}_{1}+1.5 \mathrm{~kg} \mathrm{Zn} \mathrm{ha}^{-1}$ & 3591 & 3733 & 4310 & 4538 & 4043 \\
\hline $\mathrm{T}_{4} \mathrm{~T}_{1}+3.0 \mathrm{~kg} \mathrm{Fe} \mathrm{ha}^{-1}$ & 3721 & 3863 & 4511 & 4660 & 4189 \\
\hline $\mathrm{T}_{5} \mathrm{~T}_{1}+1.5 \mathrm{~kg} \mathrm{Zn} \mathrm{ha}^{-1}+3.0 \mathrm{~kg} \mathrm{Fe} \mathrm{ha}^{-1}$ & 4159 & 4259 & 5035 & 5278 & 4682 \\
\hline $\mathrm{T}_{6} \mathrm{~T}_{1+} 0.5$ t FYM ha ${ }^{-1}$ En. with $0.75 \mathrm{~kg} \mathrm{Zn}$ & 3724 & 3911 & 4520 & 4903 & 4264 \\
\hline $\mathrm{T}_{7} \mathrm{~T}_{1+} 0.5 \mathrm{t}$ FYM ha ${ }^{-1} \mathrm{En}$. with $1.5 \mathrm{~kg} \mathrm{Fe}$ & 4014 & 4075 & 4848 & 5129 & 4516 \\
\hline $\mathrm{T}_{8} \mathrm{~T}_{1+} 0.5 \mathrm{t}$ FYM ha ${ }^{-1}$ En. with $0.75 \mathrm{~kg} \mathrm{Zn}$ and $1.5 \mathrm{~kg} \mathrm{Fe}$ & 4281 & 4396 & 5179 & 5302 & 4790 \\
\hline S.Em. \pm & 186 & 136 & 218 & 205 & 94 \\
\hline C.D. at $5 \%$ & 546 & 399 & 641 & 604 & 266 \\
\hline C.V. \% & 9.69 & 6.85 & 9.42 & 8.50 & 8.75 \\
\hline \multicolumn{6}{|l|}{ Interactions } \\
\hline $\mathbf{Y} \times \mathbf{T}$ & \multicolumn{5}{|c|}{ NS } \\
\hline
\end{tabular}


Table.9 Effect of iron and zinc enriched with FYM and without enriched FYM on straw yield of wheat

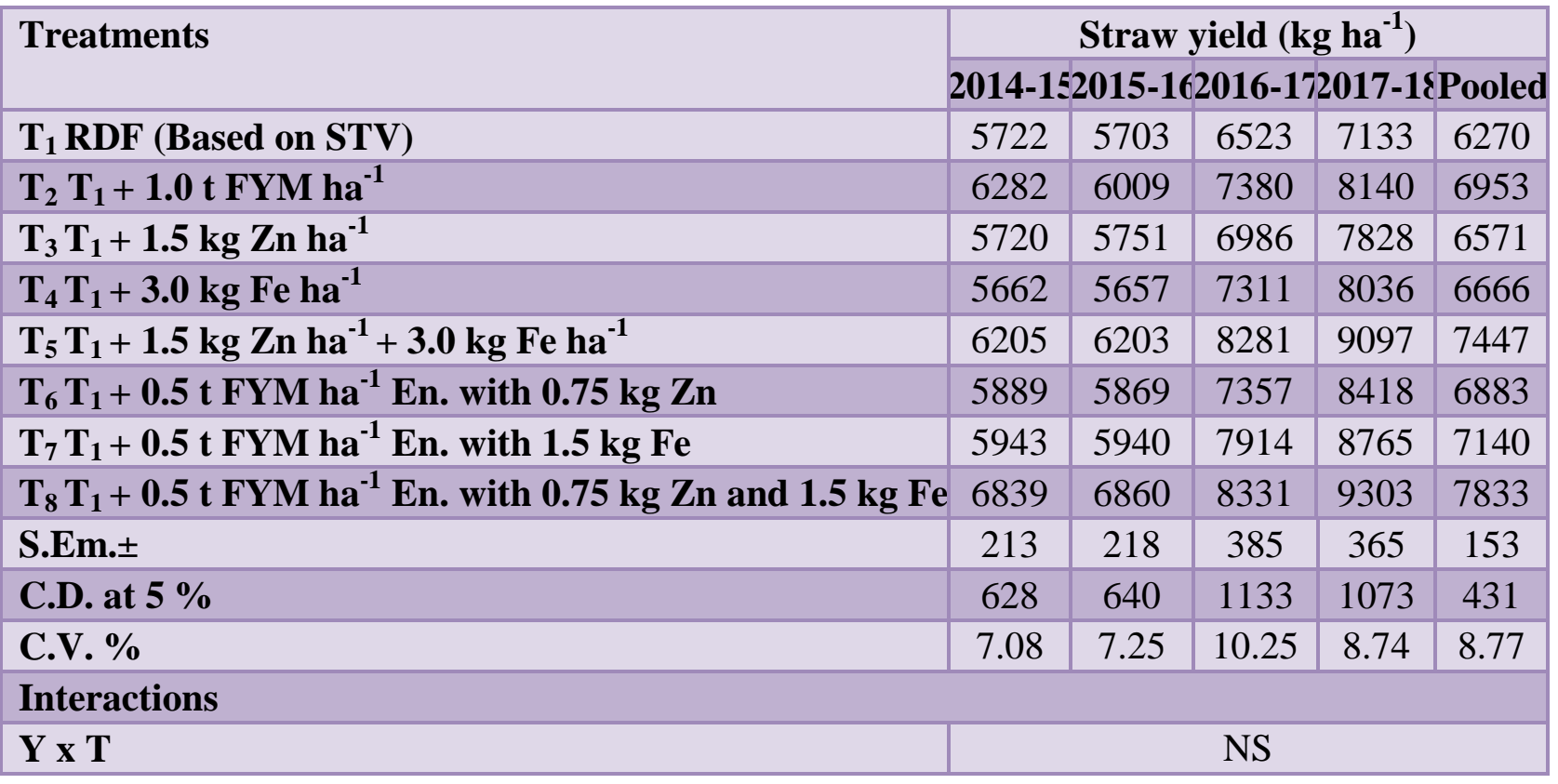

Table.10 Effect of iron and zinc enriched with FYM and without enriched FYM on the economics of wheat (Pooled data of 4 years)

\begin{tabular}{|c|c|c|c|c|c|}
\hline Treatments & $\begin{array}{c}\text { Seed } \\
\text { yield } \\
(\mathrm{kg} / \mathrm{ha})\end{array}$ & $\begin{array}{c}\begin{array}{c}\text { Cost of } \\
\text { cultivation } \\
\text { (Rs/ha) }\end{array} \\
\text { (R) }\end{array}$ & $\begin{array}{l}\text { Gross } \\
\text { returns } \\
\text { (Rs/ha) }\end{array}$ & $\begin{array}{c}\text { Net } \\
\text { returns } \\
\text { (Rs/ha) }\end{array}$ & BCR \\
\hline$T_{1}$ RDF (Based on STV) & 3781 & 30588 & 78140 & 47552 & 2.55 \\
\hline$T_{2} T_{1}+1.0$ t FYM ha ${ }^{-1}$ & 4234 & 32438 & 87372 & 54934 & 2.69 \\
\hline$T_{3} T_{1}+1.5 \mathrm{~kg} \mathrm{Zn} \mathrm{ha}^{-1}$ & 4043 & 30995 & 83291 & 52296 & 2.69 \\
\hline$T_{4} T_{1}+3.0 \mathrm{~kg} \mathrm{Fe} \mathrm{ha}^{-1}$ & 4189 & 31456 & 86010 & 54553 & 2.73 \\
\hline $\mathrm{T}_{5} \mathrm{~T}_{1}+1.5 \mathrm{~kg} \mathrm{Zn} \mathrm{ha}^{-1}+3.0 \mathrm{~kg} \mathrm{Fe} \mathrm{ha}^{-1}$ & 4682 & 31863 & 96133 & 64269 & 3.02 \\
\hline$T_{6} T_{1}+0.5$ t FYM ha $^{-1}$ En. with $0.75 \mathrm{~kg} \mathrm{Zn}$ & 4264 & 32241 & 87751 & 55509 & 2.72 \\
\hline$T_{7} T_{1}+0.5$ t FYM ha $^{-1}$ En. with $1.5 \mathrm{~kg} \mathrm{Fe}$ & 4516 & 32472 & 92640 & 60168 & 2.85 \\
\hline $\mathrm{T}_{8} \mathrm{~T}_{1}+0.5 \mathrm{t} \mathrm{FYM} \mathrm{ha}{ }^{-1}$ En. with $0.75 \mathrm{~kg} \mathrm{Zn}$ and $1.5 \mathrm{~kg} \mathrm{Fe}$ & 4790 & 32676 & 98766 & 66090 & 3.02 \\
\hline S.Em. \pm & 94 & - & - & - & - \\
\hline C.D. at $5 \%$ & 266 & - & - & - & - \\
\hline C.V. \% & 8.75 & - & - & - & - \\
\hline $\mathbf{Y} \mathbf{x} \mathbf{T}$ & NS & - & - & - & - \\
\hline
\end{tabular}

From the perusal of the data presented in Table 7, it could be inferred that application of iron alone @ $3.0 \mathrm{~kg} \mathrm{Fe} \mathrm{ha}^{-1}$ or iron and zinc enriched with FYM and without enriched
FYM showed significant increase in protein content in wheat grain over rest of the treatments on pooled basis. 


\section{Grain and straw yield}

The ultimate effect of experimental variables was reflected in the final yield of wheat crop. The consequences obtainable in Table 8 and 9 make known that application of suggested quantity of fertilizer (120-60-00 NPK $\mathrm{kg} \mathrm{ha}^{-1}$ ) on the basis of STV + 0.5 t FYM ha ${ }^{-1}$ enriched with $0.75 \mathrm{~kg} \mathrm{Zn}$ and $1.5 \mathrm{~kg} \mathrm{Fe}\left(\mathrm{T}_{8}\right)$ recorded significantly higher grain (4281, $4396,5179,5302$ and $4790 \mathrm{~kg} \mathrm{ha}^{-1}$ ) and straw (6839, 6860, 8331, 9303 and $\left.7833 \mathrm{~kg} \mathrm{ha}^{-1}\right)$ yields of wheat over $\mathrm{T}_{1}$ (Recommended dose of fertilizer on the basis of STV only) during all the individual years as well as on pooled basis. But, it was at par with $\mathrm{T}_{5}$ on pooled basis in case of grain $\left(4682 \mathrm{~kg} \mathrm{ha}^{-1}\right)$ and straw $\left(7447 \mathrm{~kg} \mathrm{ha}^{-1}\right.$ ) yield. Cakmak et al., (2008) reported that there is increasing evidence showing that foliar or combined soil foliar application of $\mathrm{Zn}$ fertilizers under field conditions are highly effective and very practical way to maximize uptake and accumulation of $\mathrm{Zn}$ in whole wheat grain, raising concentration up to $60 \mathrm{mg} \mathrm{Zn} \mathrm{kg}{ }^{-1}$. Increase in yield was due to improved availability of iron and zinc which could be attributed to the formation of stable organometalic complexes with organic matter, especially during the enrichment process to last for a longer time and release the nutrients slowly in the soil system in such a way that the nutrients are protected from fixation and made available to the plant root system throughout the crop growth (Meena et al., 2006).

The soil application of RDF (120-60-00 NPK $\mathrm{kg} \mathrm{ha}^{-1}$ ) on the basis of STV + 0.5 t FYM ha ${ }^{-1}$ enriched with $0.75 \mathrm{~kg} \mathrm{Zn}$ and $1.5 \mathrm{~kg} \mathrm{Fe}$ realized significantly higher gross returns $\left(98,766 \mathrm{ha}^{-1}\right)$, net returns $\left(66,090 \mathrm{ha}^{-1}\right)$ and $\mathrm{BC}$ ratio (3.02) as compared to other treatments which was mainly due to higher grain and straw yield compared to others. Significantly, the lower gross returns $(78,140$ $\left.\mathrm{ha}^{-1}\right)$, net returns $\left(47,552 \mathrm{ha}^{-1}\right)$ and $\mathrm{BC}$ ratio (2.55) were obtained in control compared to other treatments which was due to lower grain and straw yield of wheat (Table 10). Based on the results, it was concluded that soil application of RDF (120-60-00 NPK kg ha ${ }^{-1}$ ) on the basis of STV + 0.5 t FYM ha ${ }^{-1}$ enriched with $0.75 \mathrm{~kg} \mathrm{Zn}$ and $1.5 \mathrm{~kg}$ Fe to wheat was found optimum for getting higher grain yield, straw yield and also higher net returns compared to recommended dose of fertilizer on the basis of STV only.

\section{References}

Anonymous. Annual Report, (2017). Directorate of Economics \& Statistics, Department of Agriculture, Cooperation \& Farmers Welfare, New Delhi.

Meena, M. C., Patel, K. P. and Rathod, D. D. (2006). Effect of $\mathrm{Zn}$ and $\mathrm{Fe}$ enriched FYM on mustard yield and micronutrients availability in loamy sand soil of Anand. J. Indian Soc. Soil Sci., 54: 495-499.

Cakmak, I., (2008). Enrichment of cereal grains with zinc: agronomic or genetic biofortification. Plant and Soil. 302(1\&2):1-17.

Shivay, Y.S., Prasad, R., Shukla, A.K., Das, S. (2014). Zinc Management" Text Book of Plant Nutrient Management by Prasad et al., First Edition. 182187.

Cakmak, I., and Kutman, U.B. (2017). Agronomic biofortification of cereals with zinc: a review. European Journal of Soil Science, 69(1): 172-180.

Navrang, S. and Tomar, G.S. (2016). Effect of integrated use of FYM and urea on yield, nutrient uptake and protein content of wheat (Triticum aestivum L.). Supplement on Agronomy, 11(1): 663-668.

Gul, H., Said, A., Saeed, B., Fida, M. and 
Ahmad, I., (2011). Effect of foliar application of nitrogen, potassium and zinc on wheat growth. Journal of
Agricultural and Biological Science, 6(4): 56-58.

\section{How to cite this article:}

Malav, J.K., N.N. Salvi, J.K. Patel, J.R. Jat, S. Kumar, R.P. Pavaya, B.T. Patel and Patel, V.R. 2019. Effect of Iron and Zinc Enriched FYM on Growth, Yield and Quality of Wheat (Triticum aestivum L) in Salt Affected Soils of Gujarat. Int.J.Curr.Microbiol.App.Sci. 8(06): 2960-2969. doi: https://doi.org/10.20546/ijcmas.2019.806.354 